\title{
The Synthesis of Medium-Chain-Length $\beta$-Hydroxy Esters via the Reformatsky Reaction
}

\author{
Miloslav Sailer \\ Krystyn I. Dubicki \\ John L. Sorensen* \\ Department of Chemistry, University of Manitoba, Winnipeg, \\ MB, R3T 2N2, Canada \\ John.Sorensen@umanitoba.ca
}

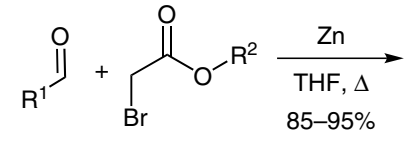<smiles>[R]OC(=O)CC([R])O</smiles>

$\mathrm{R}^{1}=\left(\mathrm{CH}_{2}\right)_{n} \mathrm{Me} ; \mathrm{n}=2-8$ $\mathrm{R}^{2}=\mathrm{Me}, \mathrm{Et}$
Received: 09.07.2014

Accepted after revision: 24.09.2014

Published online: 29.10 .2014

DOI: 10.1055/s-0034-1379479; Art ID: ss-2014-m0434-op

Abstract The synthesis of medium-chain-length $\beta$-hydroxy esters in good yield via the Reformatsky reaction is described. This work will be used as the basis for further investigation of hydroxyalkanoate polymers as potential feedstock for biofuel production.

Key words Reformatsky reaction, biosynthesis, polyhydroxyalkanoate, biofuel, 3-hydroxy esters, Pseudomonas putida

There has been recent interest in developing alternate sources of biofuels that can be used as a replacement for fossil fuels. One of the most prevalent biofuels is biodiesel produced by the methanolysis of long-chain (C16-C20) fatty acid triacyl glycerides that are the major constituents of plant oils. ${ }^{1}$ However, biodiesel production results in several by-products, such as glycerol and free fatty acids, which cannot be used as fuels and are of otherwise low value. It has been recently demonstrated that these waste by-products can be used as feedstock for the bacteria Pseudomonas putida LS46. ${ }^{2}$ This strain of $P$. putida can efficiently convert these by-products into a variety of medium-chain-length polyhydroxyalkanoates (mcl-PHAs - Scheme 1), with chain lengths of 6 to 14 carbons. These bioester polymers can be considered of higher value than the biodiesel waste byproducts. For example, polyhydroxyalkanoate (PHA) has been investigated as a feedstock for biodegradable plastics and other products. ${ }^{3}$

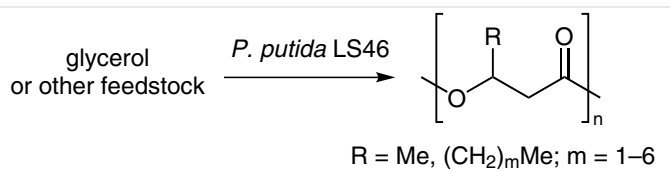

Scheme 1 The production of polyhydroxyalkanoates from glycerol or other feedstock by $P$. putida LS46
We are interested in investigating the chemical conversion of PHA into other value-added products, such as 'dropin' biofuels. For example the methanolysis of PHA results in a 3-hydroxymethyl ester with the chain length, and resulting chemical and physical properties, which is dependent on the chemical composition of the feedstock polymer. We decided to investigate 3-hydroxymethyl and -ethyl esters with a full range of chain lengths to determine the optimal of carbon atoms for downstream conversion to biofuel. However, optimizing growth conditions for P. putida to produce PHA with a specific chain length is time-consuming and expensive. In addition, although the free acids are commercially available their average cost $(\sim 10 / \mathrm{mg})$ requires the development of a more economical synthesis. Therefore, a synthetic methodology was developed that would provide a convenient and economical access to a series of 3hydroxy esters of the required chain length (C4-C12).

We decided to investigate the use of the Reformatsky reaction for this purpose as it is one of the most useful methods for the formation 3-hydroxy esters. ${ }^{4}$ The Reformatsky reaction can be carried out in aqueous neutral conditions, in contrast to the alkaline conditions required for aldol condensations or the dry inert conditions required when using Grignard reagents. The Reformatsky reaction has been extended to a large variety of substrates ${ }^{5}$ and an asymmetric version has even been developed. ${ }^{6}$ It was decided that this reaction would offer an attractive approach to synthesize our desired compounds as a series of aldehyde precursors are commercially available as is both ethyl and methyl bromoacetate. Here, we report the synthesis of a series of 3-hydroxymethyl and -ethyl esters in good yields using the Reformatsky reaction.

The Reformatsky reaction was used to generate the $\beta$ hydroxy esters (Table 1 ) reported here. The reactions were carried out using wet THF as solvent since it had been previously reported that the use of wet THF in the Reformatsky 
reaction produces significantly better yields with aliphatic aldehydes than anhydrous THF. ${ }^{7}$ Preliminary method development reactions were conducted on a small scale (2 $\mathrm{mmol}$ ) with the product yields ranging from 42 to $81 \%$. Slow addition of $\mathrm{BF}_{3} \cdot \mathrm{OEt}_{2}$ via syringe pump was also used.

Table 1 Synthesis of C6 to C12 $\beta$-Hydroxy Esters

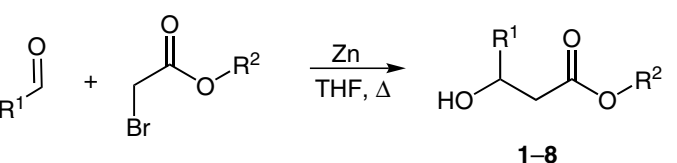

\begin{tabular}{llll}
\hline Product & $\mathrm{R}^{1}$ & $\mathrm{R}^{2}$ & Yield (\%) \\
\hline $\mathbf{1}$ & $\left(\mathrm{CH}_{2}\right)_{2} \mathrm{Me}$ & $\mathrm{Me}$ & 90 \\
$\mathbf{2}$ & $\left(\mathrm{CH}_{2}\right)_{2} \mathrm{Me}$ & $\mathrm{Et}$ & 90 \\
$\mathbf{3}$ & $\left(\mathrm{CH}_{2}\right)_{4} \mathrm{Me}$ & $\mathrm{Me}$ & 85 \\
$\mathbf{4}$ & $\left(\mathrm{CH}_{2}\right)_{4} \mathrm{Me}$ & $\mathrm{Et}$ & 86 \\
$\mathbf{5}$ & $\left(\mathrm{CH}_{2}\right)_{6} \mathrm{Me}$ & $\mathrm{Me}$ & 92 \\
$\mathbf{6}$ & $\left(\mathrm{CH}_{2}\right)_{6} \mathrm{Me}$ & $\mathrm{Et}$ & 93 \\
$\mathbf{7}$ & $\left(\mathrm{CH}_{2}\right)_{8} \mathrm{Me}$ & $\mathrm{Me}$ & 94 \\
$\mathbf{8}$ & $\left(\mathrm{CH}_{2}\right)_{8} \mathrm{Me}$ & $\mathrm{Et}$ & 95 \\
\hline
\end{tabular}

In order to produce an amount of each 3-hydroxy ester sufficient for testing as potential biofuels we decided to optimize the reaction at a larger scale. However, due to the exothermic nature of the Reformatsky reaction, scale-up often requires specialized equipment and reaction conditions. ${ }^{8,9}$ We therefore decided to optimize our reaction at the 0.1 mole scale as it was felt that this would prevent runaway reactions, which may occur at larger scales. Initial attempts at this scale involved the formation of the zinc enolate through slow addition of bromoacetate to a mixture of $\mathrm{BF}_{3} \cdot \mathrm{OEt}_{2}$-activated zinc. This was followed by slow addition of a solution of the aldehyde to this mixture. Although runaway reactions did not occur, a mixture of products was observed irrespective of addition rate. All attempts to optimize the reaction through changing order and rate of addition of reagents resulted in complex mixtures with low yields of desired product.

As part of the optimization process, we discovered that it was not necessary to activate the zinc granules with $\mathrm{BF}_{3} \cdot \mathrm{OEt}_{2}$ if they were suspended in refluxing THF. Therefore, in order to minimize side products, the following procedure was developed. To a refluxing solution of THF were added zinc ( 2 equiv), aldehyde, and bromoacetate ( 2 equiv) in quick succession. WARNING: This reaction requires venting as the mixture immediately undergoes rapid solvent boiling, which requires extensive condensation. However, under these conditions the reaction is complete in 20 minutes with high yields (85-95\%). The yields of the 3-hydroxy esters produced by the Reformatsky reactions under these conditions are summarized in Table 1.
In order to complete our library of molecules for biofuel testing, the 3-hydroxy esters were hydrolyzed to the corresponding free acids under basic conditions. These results are summarized in Table 2. These free acids are oils or solids at room temperature and therefore may not be suitable as biofuels. However, they may serve as useful substrates for deoxygenation reactions in the downstream conversion of the $P$. putida polyhydroxyalkanoates.

Table 2 Saponification of $\beta$-Hydroxy Esters to $\beta$-Hydroxy Acids

\begin{tabular}{lll} 
& \\
\hline Product & $\mathrm{R}^{1}$ & Yield (\%) \\
\hline $\mathbf{9}$ & $\left(\mathrm{CH}_{2}\right)_{2} \mathrm{Me}$ & 72 \\
$\mathbf{1 0}$ & $\left(\mathrm{CH}_{2}\right)_{4} \mathrm{Me}$ & 81 \\
$\mathbf{1 1}$ & $\left(\mathrm{CH}_{2}\right)_{6} \mathrm{Me}$ & 92 \\
$\left(\mathrm{CH}_{2}\right)_{8} \mathrm{Me}$ & 86
\end{tabular}

In summary, we have synthesized a series of 3-hydroxy esters with carbon chain lengths of 6-12 carbons using the Reformatsky reaction. This method has been optimized for an intermediate scale of $100 \mathrm{mmol}$, which prevents runaway reactions and the formation of side products. This intermediate scale also provides an amount of product that is sufficient for evaluating these molecules as potential biofuels. This testing is underway and will be reported on when complete.

All ${ }^{1} \mathrm{H}$ NMR and ${ }^{13} \mathrm{C}$ NMR spectra were obtained using a Bruker AC 300 in $\mathrm{CDCl}_{3}$ unless otherwise stated. IR spectra were obtained by ATR on a Bruker Alpha FT-IR using a thin film formed by solvent evaporation.

\section{Reformatsky Reaction; General Procedure}

THF (200 mL) was added to an oven-dried $500 \mathrm{~mL}$ round-bottomed flask equipped with a condenser $30 \mathrm{~cm}$ in length and left open to the atmosphere. The THF was used directly as purchased without any further drying or purification. The solvent was then rapidly stirred using a magnetic stir bar and brought to reflux $\left(66{ }^{\circ} \mathrm{C}\right)$ on a sand bath. The condenser was then temporarily raised and $6.5 \mathrm{~g}$ ( $0.1 \mathrm{~mol}, 2$ equiv) of $\mathrm{Zn}$ granules were added to the hot solvent. This was followed by the rapid addition of the aldehyde $(0.1 \mathrm{~mol})$ and the bromoacetate (as either the methyl or ethyl ester) ( $0.2 \mathrm{~mol}, 2$ equiv) into the reaction mixture. The condenser was immediately reattached to the roundbottomed flask at which point rapid boiling occurred. The reaction was allowed to rapidly reflux for $1 \mathrm{~min}$ at which point the reaction vessel was removed from heat and allowed to cool to r.t. with stirring. The reaction was observed to be complete by TLC (eluent: hexanesEtOAc, 80:20) within 20 min for all substrates. Excess THF was removed under vacuum and the resultant brown oil was dissolved in hexanes and quenched with $\mathrm{H}_{2} \mathrm{O}$ to form a yellow precipitate. The mixture was filtered and the hexane layer was washed with $\mathrm{H}_{2} \mathrm{O}$ (100 
$\mathrm{mL})$, aq $1 \mathrm{M} \mathrm{HCl}(2 \times 50 \mathrm{~mL}), \mathrm{H}_{2} \mathrm{O}(100 \mathrm{~mL})$ and brine $(50 \mathrm{~mL})$, and dried $\left(\mathrm{Na}_{2} \mathrm{SO}_{4}\right)$. Removal of hexanes under vacuum furnished the products 1-8 in yields ranging from 86 to 95\% (Table 1).

\section{Methyl 3-Hydroxyhexanoate (1)}

Yield: $13.14 \mathrm{~g}$ (90\%, $90 \mathrm{mmol})$; clear yellow oil.

IR (film): 3468w, 2957m, 1724s, 1437m, 1166s, 1122m, 993m, 847w $\mathrm{cm}^{-1}$.

${ }^{1} \mathrm{H}$ NMR $\left(300 \mathrm{MHz}, \mathrm{CDCl}_{3}\right): \delta=0.91(\mathrm{t}, J=7.3 \mathrm{~Hz}, 3 \mathrm{H}), 1.42(\mathrm{~m}, 4 \mathrm{H})$, $2.38(\mathrm{dd}, J=9.1,16.9 \mathrm{~Hz}, 1 \mathrm{H}), 2.49(\mathrm{dd}, J=4.2,16.3 \mathrm{~Hz}, 1 \mathrm{H}), 2.90(\mathrm{br}$ s, $1 \mathrm{H}), 3.69$ (s, $3 \mathrm{H}), 3.99(\mathrm{~m}, 1 \mathrm{H})$.

${ }^{13} \mathrm{C} \mathrm{NMR}\left(75 \mathrm{MHz}, \mathrm{CDCl}_{3}\right): \delta=14.0,18.7,38.7,41.2,51.8,67.8,173.5$.

\section{Ethyl 3-Hydroxyhexanoate (2)}

Yield: $14.4 \mathrm{~g}$ (90\%, $90 \mathrm{mmol})$; clear yellow oil.

IR (film): 3459w, 2960w, 1718s, 1465w, 1372m, 1166s, 1017s, 847w, $733 \mathrm{w} \mathrm{cm}^{-1}$.

${ }^{1} \mathrm{H}$ NMR $\left(300 \mathrm{MHz}, \mathrm{CDCl}_{3}\right): \delta=0.91(\mathrm{t}, J=7.0 \mathrm{~Hz}, 3 \mathrm{H}), 1.25(\mathrm{t}, J=7.6$ $\mathrm{Hz}, 3 \mathrm{H}), 1.41(\mathrm{~m}, 4 \mathrm{H}), 2.37$ (dd, $J=9.1,16.7 \mathrm{~Hz}, 1 \mathrm{H}), 2.47$ (dd, $J=3.4$, $17.3 \mathrm{~Hz}, 1 \mathrm{H}), 2.96(\mathrm{~d}, J=3.78 \mathrm{~Hz}, 1 \mathrm{H}), 3.99(\mathrm{~m}, 1 \mathrm{H}), 4.14(\mathrm{q}, J=7.0$ $\mathrm{Hz}, 2 \mathrm{H})$.

${ }^{13} \mathrm{C}$ NMR $\left(75 \mathrm{MHz}, \mathrm{CDCl}_{3}\right): \delta=14.0,14.2,18.7,38.7,41.4,60.7,67.8$, 173.2.

\section{Methyl 3-Hydroxyoctanoate (3)}

Yield: $14.79 \mathrm{~g}$ (85\%, $85 \mathrm{mmol})$; clear yellow oil.

IR (film): 3458w, 2929m, 1724s, $1437 \mathrm{~m}, 1164 \mathrm{~s} \mathrm{~cm}^{-1}$.

${ }^{1} \mathrm{H} \mathrm{NMR}\left(300 \mathrm{MHz}, \mathrm{CDCl}_{3}\right): \delta=0.88(\mathrm{t}, J=6.3 \mathrm{~Hz}, 3 \mathrm{H}), 1.29(\mathrm{~m}, 8 \mathrm{H})$, 2.40 (dd, $J=8.8,16.9 \mathrm{~Hz}, 1 \mathrm{H}$ ), 2.51 (dd, $J=3.8,15.6 \mathrm{~Hz}, 1 \mathrm{H}$ ), 3.70 (s, 3 $\mathrm{H}), 3.99$ ( $\mathrm{m}, 1 \mathrm{H})$.

${ }^{13} \mathrm{C}$ NMR $\left(75 \mathrm{MHz}, \mathrm{CDCl}_{3}\right): \delta=14.1,22.7,25.2,31.8,36.6,41.2,51.8$, 68.1, 173.6.

\section{Ethyl 3-Hydroxyoctanoate (4)}

Yield: $16.17 \mathrm{~g}$ (86\%, $86 \mathrm{mmol})$; clear yellow oil.

IR (film): 3432w, 2931m, 1718s, 1372m, 1162s, 1026s, 732 $\mathrm{w} \mathrm{cm}^{-1}$.

$\left.{ }^{1} \mathrm{H} \mathrm{NMR} \mathrm{(300} \mathrm{MHz,} \mathrm{CDCl} 3\right): \delta=0.88(\mathrm{t}, J=6.7 \mathrm{~Hz}, 3 \mathrm{H}), 1.27(\mathrm{~m}, 11 \mathrm{H})$, $2.39(\mathrm{dd}, J=8.82,16.5 \mathrm{~Hz}, 1 \mathrm{H}), 2.50(\mathrm{dd}, J=3.9,15.8 \mathrm{~Hz}, 1 \mathrm{H}), 2.90(\mathrm{~s}$, $1 \mathrm{H}), 3.99(\mathrm{~m}, 1 \mathrm{H}), 4.17(\mathrm{q}, J=7.0 \mathrm{~Hz}, 2 \mathrm{H})$.

${ }^{13} \mathrm{C}$ NMR $\left(75 \mathrm{MHz}, \mathrm{CDCl}_{3}\right): \delta=14.1,14.3,22.7,25.3,31.8,36.6,41.4$, $60.8,68.2,173.2$.

\section{Methyl 3-Hydroxydecanoate (5)}

Yield: $18.58 \mathrm{~g}$ (92\%, $92 \mathrm{mmol})$; clear yellow oil.

IR (film): 3478w, 2925s, 2855m, 1726s, 1437m, 1163s, 1056m, 991s, $723 \mathrm{w} \mathrm{cm}^{-1}$.

${ }^{1} \mathrm{H} \mathrm{NMR}\left(300 \mathrm{MHz}, \mathrm{CDCl}_{3}\right): \delta=0.87(\mathrm{t}, J=6.5 \mathrm{~Hz}, 3 \mathrm{H}), 1.42(\mathrm{~m}, 12 \mathrm{H})$, $2.40(\mathrm{dd}, J=8.8,16.3 \mathrm{~Hz}, 1 \mathrm{H}), 2.51(\mathrm{dd}, J=3.3,16.4 \mathrm{~Hz}, 1 \mathrm{H}), 2.84(\mathrm{br}$ $\mathrm{s}, 1 \mathrm{H}), 3.71(\mathrm{~s}, 3 \mathrm{H}), 3.99(\mathrm{~m}, 1 \mathrm{H})$.

${ }^{13} \mathrm{C}$ NMR $\left(75 \mathrm{MHz}, \mathrm{CDCl}_{3}\right): \delta=14.2,22.7,25.6,29.3,29.6,31.9,36.6$, $41.2,51.8,68.2,173.6$.

\section{Ethyl 3-Hydroxydecanoate (6)}

Yield: $20.1 \mathrm{~g}$ (93\%, $93 \mathrm{mmol})$; clear yellow oil.

IR (film): 3496w, 2927m, 1720m, 907s, 728s, $647 \mathrm{~m} \mathrm{~cm}^{-1}$.
${ }^{1} \mathrm{H}$ NMR $\left(300 \mathrm{MHz}, \mathrm{CDCl}_{3}\right): \delta=0.87(\mathrm{t}, J=6.7 \mathrm{~Hz}, 3 \mathrm{H}), 1.27(\mathrm{~m}, 15 \mathrm{H})$, 2.38 (dd, $J=9.0,16.3 \mathrm{~Hz}, 1 \mathrm{H}$ ), 2.50 (dd, $J=3.5,16.1 \mathrm{~Hz}, 1 \mathrm{H}$ ), 2.95 (br, $1 \mathrm{H}), 3.99(\mathrm{~m}, 1 \mathrm{H}), 4.16$ (q, J = 7.0 Hz, $2 \mathrm{H}$ ).

${ }^{13} \mathrm{C}$ NMR $\left(75 \mathrm{MHz}, \mathrm{CDCl}_{3}\right): \delta=14.2,14.3,22.7,25.6,29.3,29.6,31.9$, 36.6, 41.4, 60.8, 68.2, 173.2 .

\section{Methyl 3-Hydroxydodecanoate (7)}

Yield: $21.62 \mathrm{~g}$ (94\%, $94 \mathrm{mmol})$; clear yellow oil. IR (film): 3468w, 2923s, 2853m, 1726s, 1437m, 1170s, 732m cm-1. ${ }^{1} \mathrm{H} \mathrm{NMR}\left(300 \mathrm{MHz}, \mathrm{CDCl}_{3}\right): \delta=0.88(\mathrm{t}, J=5.9 \mathrm{~Hz}, 3 \mathrm{H}), 1.28(\mathrm{~m}, 16 \mathrm{H})$, 2.45 (dd, $J=16.2,18.8 \mathrm{~Hz}, 1 \mathrm{H}$ ), 2.47 (dd, $J=13.2,16.2 \mathrm{~Hz}, 1 \mathrm{H}$ ), 2.99 (br, $1 \mathrm{H}), 3.70(\mathrm{~s}, 3 \mathrm{H}), 4.00(\mathrm{~m}, 1 \mathrm{H})$.

${ }^{13} \mathrm{C}$ NMR $\left(75 \mathrm{MHz}, \mathrm{CDCl}_{3}\right): \delta=14.1,22.7,25.5,29.3,29.5,29.5$ 29.6, $31.9,36.6,41.2,51.7,68.0,173.5$.

\section{Ethyl 3-Hydroxydodecanoate (8)}

Yield: $23.18 \mathrm{~g}$ (95\%, $95 \mathrm{mmol})$; clear yellow oil. IR (film): 3496w, 2927m, 1720m, 907s, 728s, $647 \mathrm{~m} \mathrm{~cm}^{-1}$.

${ }^{1} \mathrm{H} \mathrm{NMR}\left(300 \mathrm{MHz}, \mathrm{CDCl}_{3}\right): \delta=0.87(\mathrm{t}, J=7.0 \mathrm{~Hz}, 3 \mathrm{H}), 1.26(\mathrm{~m}, 19 \mathrm{H})$, 2.43 (dd, $J=15.9,19.0 \mathrm{~Hz}, 1 \mathrm{H}), 2.45$ (dd, $J=13.5,16.6 \mathrm{~Hz}, 1 \mathrm{H}$ ), 3.05 (br, $1 \mathrm{H}), 3.99$ (m, $1 \mathrm{H}), 4.16(\mathrm{q}, J=7.0 \mathrm{~Hz}, 2 \mathrm{H})$.

${ }^{13} \mathrm{C}$ NMR $\left(75 \mathrm{MHz}, \mathrm{CDCl}_{3}\right): \delta=14.1,14.2,22.7,25.5,29.3,29.5,29.5$, 29.6, 31.9, 36.6, 41.4, 60.6, 68.0, 173.1.

\section{Saponification of Esters; General Procedure}

To a $50 \mathrm{~mL}$ round-bottomed flask fitted with a stir bar was added hexane $(5 \mathrm{~mL})$ and the respective 3-hydroxymethyl ester $(1 \mathrm{mmol})$. This mixture was heated to $50^{\circ} \mathrm{C}$ at which point sat. $\mathrm{KOH}$ in $\mathrm{MeOH}(0$. $5 \mathrm{~mL}$ ) was added. This led to the instant formation of a precipitate. The mixture was then stirred vigorously at $60{ }^{\circ} \mathrm{C}$ for $30 \mathrm{~min}$, then removed from heat, and the solvents were evaporated under reduced pressure to provide the product as a potassium salt. This residue was dissolved in distilled $\mathrm{H}_{2} \mathrm{O}(10 \mathrm{~mL})$ and extracted with $\mathrm{CHCl}_{3}(3 \times 10$ $\mathrm{mL}$ ). The aqueous layer was collected and acidified with concd $\mathrm{HCl}$ to $\mathrm{pH}<1 . \mathrm{Et}_{2} \mathrm{O}(20 \mathrm{~mL})$ was added to the aqueous acidic solution and this solution was stirred vigorously for $1 \mathrm{~h}$. The organic and aqueous layers were separated the aqueous layer was extracted with $\mathrm{Et}_{2} \mathrm{O}(2 \times 20$ $\mathrm{mL})$. The combined organic layers were dried $\left(\mathrm{Na}_{2} \mathrm{SO}_{4}\right)$, filtered, and evaporated under reduced pressure to give the corresponding product, which was used without further purification (Table 2).

\section{3-Hydroxyhexanoic Acid (9)}

Yield: $95 \mathrm{mg}$ (72\%, $0.72 \mathrm{mmol})$; clear oil.

IR (film): 3390br, 2959m, 1704s, 1407m, 1174m, 1123m, 1017m, $845 \mathrm{~m} \mathrm{~cm}^{-1}$.

${ }^{1} \mathrm{H}$ NMR $\left(300 \mathrm{MHz}, \mathrm{CDCl}_{3}\right): \delta=0.90(\mathrm{t}, J=7.30 \mathrm{~Hz}, 3 \mathrm{H}), 1.43(\mathrm{~m}, 4 \mathrm{H})$, $2.43(\mathrm{dd}, J=8.76,16.4 \mathrm{~Hz}, 1 \mathrm{H}), 2.53(\mathrm{dd}, J=4.57,16.3 \mathrm{~Hz}, 1 \mathrm{H}), 4.03$ (m, $1 \mathrm{H}), 7.20$ (br, $1 \mathrm{H})$.

${ }^{13} \mathrm{C} \mathrm{NMR}\left(75 \mathrm{MHz}, \mathrm{CDCl}_{3}\right): \delta=13.9,18.7,38.6,41.2,68.0,177.4$.

\section{3-Hydroxyoctanoic Acid (10)}

Yield: $130 \mathrm{mg}$ (81\%, $0.81 \mathrm{mmol})$; yellow oil.

IR (film): 3312br, 2929m, 1707s, 1374w, 1238m, 1044m, 933w, 872w, $608 \mathrm{w} \mathrm{cm}^{-1}$.

${ }^{1} \mathrm{H} \mathrm{NMR}\left(300 \mathrm{MHz}, \mathrm{CDCl}_{3}\right): \delta=0.88(\mathrm{t}, J=6.76 \mathrm{~Hz}, 3 \mathrm{H}), 1.30(\mathrm{~m}, 8 \mathrm{H})$, 2.46 (dd, $J=9.01,16.3 \mathrm{~Hz}, 1 \mathrm{H}), 2.56$ (dd, $J=4.21,16.0 \mathrm{~Hz}, 1 \mathrm{H}$ ), 4.03 (m, $1 \mathrm{H}), 6.42$ (br, $1 \mathrm{H})$. 
${ }^{13} \mathrm{C}$ NMR $\left(75 \mathrm{MHz}, \mathrm{CDCl}_{3}\right): \delta=14.1,22.7,25.2,31.7,36.5,41.2,68.2$, 177.9.

\section{3-Hydroxydecanoic Acid (11)}

Yield: $173 \mathrm{mg}$ (92\%, $0.92 \mathrm{mmol})$; white solid; $\mathrm{mp} 57.5^{\circ} \mathrm{C}$.

IR (film): 3534w ( $\left.\mathrm{H}_{2} \mathrm{O}\right), 3036 \mathrm{br}, 2920 \mathrm{~s}, 2848 \mathrm{~m}, 1679 \mathrm{~s}, 1439 \mathrm{~m}, 1221 \mathrm{~s}$, 907m, 710w, 544 $\mathrm{w} \mathrm{cm}^{-1}$.

${ }^{1} \mathrm{H} \mathrm{NMR}\left(300 \mathrm{MHz}, \mathrm{CDCl}_{3}\right): \delta=0.87(\mathrm{t}, J=6.61 \mathrm{~Hz}, 3 \mathrm{H}), 1.27(\mathrm{~m}, 12 \mathrm{H})$, 2.45 (dd, $J=8.92,16.6 \mathrm{~Hz}, 1 \mathrm{H}), 2.56$ (dd, $J=3.84,16.4 \mathrm{~Hz}, 1 \mathrm{H}), 4.03$ (m, $1 \mathrm{H}), 6.68$ (br, $1 \mathrm{H})$.

${ }^{13} \mathrm{C}$ NMR $\left(75 \mathrm{MHz}, \mathrm{CDCl}_{3}\right): \delta=14.2,22.7,25.5,29.3,29.5,31.9,36.6$, 41.2, 68.2, 178.0.

\section{3-Hydroxydodecanoic Acid (12)}

Yield: $186 \mathrm{mg}(86 \%, 0.86 \mathrm{mmol})$; white solid; $\mathrm{mp} 74{ }^{\circ} \mathrm{C}$.

IR (film): 3534w ( $\left.\mathrm{H}_{2} \mathrm{O}\right), 2952 \mathrm{br}, 2913 \mathrm{~s}, 2847 \mathrm{~m}, 1680 \mathrm{~s}, 1469 \mathrm{w}, 1441 \mathrm{w}$, $1216 \mathrm{~m}, 866 \mathrm{w}, 548 \mathrm{~m} \mathrm{~cm}^{-1}$.

${ }^{1} \mathrm{H}$ NMR (300 MHz, acetone- $\left.d_{6}\right): \delta=0.87(\mathrm{t}, J=6.34 \mathrm{~Hz}, 3 \mathrm{H}), 1.29(\mathrm{~m}$, $16 \mathrm{H}), 2.36$ (dd, $J=8.02,15.6 \mathrm{~Hz}, 1 \mathrm{H}), 2.45(\mathrm{dd}, J=4.66,15.6 \mathrm{~Hz}, 1 \mathrm{H}$ ), 2.80 (br, $1 \mathrm{H}), 3.97(\mathrm{~m}, 1 \mathrm{H})$.

\section{Acknowledgment}

Funding from the BioFuelNet Network of Centers of Excellence and the Manitoba Centers of Excellence Fund is gratefully acknowledged.

\section{Supporting Information}

Supporting information for this article is available online at http://dx.doi.org/10.1055/s-0034-1379479. Included are ${ }^{1} \mathrm{H}$ and ${ }^{13} \mathrm{C}$ NMR spectra.

\section{References}

(1) Issariyakul, T.; Dalai, A. K. Renew. Sust. Energ. Rev. 2014, 31, 446.

(2) Sharman, K. P.; Fu, J.; Cicek, N.; Sparling, R.; Levin, B. D. Can. J. Microbiol. 2012, 58, 982.

(3) Yates, M. R.; Barlow, C. Y. Resour. Conserv. Recycl. 2013, 78, 54.

(4) (a) Ocampo, R.; Dolbier, R. W. Jr. Tetrahedron 2004, 60, 9325. (b) Fürstner, A. Synthesis 1989, 571.

(5) Orsini, F.; Sello, G. Curr. Org. Synth. 2004, 1, 111.

(6) (a) Marcotte, S.; Pannecoucke, X.; Feasson, C.; Quirion, J. J. Org. Chem. 1999, 64, 8461. (b) Sorochinsky, A.; Voloshin, N.; Markovsky, A.; Belik, M.; Yasuda, N.; Uekusa, H.; Ono, T.; Berbasov, O. D.; Soloshonok, V. A. J. Org. Chem. 2003, 68, 7448.

(7) Chattopadhyay, A.; Salaskar, A. Synthesis 2000, 561.

(8) Girgis, J. M.; Liang, K. J.; Du, Z.; Slade, J.; Prasad, K. Org. Process Res. Dev. 2009, 13, 1094.

(9) Loh, G.; Tanigawara, R.; Shaik, M. S.; Sa-ei, K.; Wong, L.; Sharratt, N. P. Org. Process Res. Dev. 2012, 16, 958.

This article differs from the e-first online version only in its layout; no content has been changed. 